\title{
Géolinguistique
}

$20 \mid 2020$

Varia

\section{Notes sur la distribution géographique de certaines formes pronominales atones dans les parlers des Alpes-Maritimes (PAM)}

Notes on the Geographic Distribution of Certain Unstressed Pronominal Forms in Alpes-Maritimes Varieties

Robert Mooney et David Heap

\section{(2penEdition \\ Journals}

Édition électronique

URL : http://journals.openedition.org/geolinguistique/2352

DOI : 10.4000/geolinguistique.2352

ISSN : 2650-8176

\section{Éditeur}

UGA Éditions/Université Grenoble Alpes

\section{Édition imprimée}

ISBN : 978-2-37747-246-8

ISSN : 0761-9081

\section{Référence électronique}

Robert Mooney et David Heap, « Notes sur la distribution géographique de certaines formes pronominales atones dans les parlers des Alpes-Maritimes (PAM) », Géolinguistique [En ligne], 20 | 2020, mis en ligne le 01 décembre 2020, consulté le 19 janvier 2021. URL : http:// journals.openedition.org/geolinguistique/2352 ; DOI : https://doi.org/10.4000/geolinguistique.2352

Ce document a été généré automatiquement le 19 janvier 2021.

Géolinguistique 
Notes sur la distribution géographique de certaines formes pronominales atones dans les parlers des Alpes-Maritimes (PAM) ${ }^{1}$

Notes on the Geographic Distribution of Certain Unstressed Pronominal Forms in Alpes-Maritimes Varieties

Robert Mooney et David Heap

\section{Remerciements}

Nous remercions la professeure Michèle Oliviéri et le Dr Philippe Del Giudice de l'Université Nice Sophia-Antipolis / CNRS, UMR 6039, « Bases, corpus, langage ».

\section{Introduction}

Dans ce travail, nous examinons les pronoms atones objets des variétés romanes des localités qui se trouvent dans le département des Alpes-Maritimes afin de mieux comprendre la distribution géographique des systèmes pronominaux dans les dialectes. Nous documentons également les formes syncrétiques ainsi qu'une forme opaque observée dans la partie occidentale du département.

2 Nous identifions trois systèmes pronominaux que nous désignons ici, par des désignations de convenance, comme le maritime élargi (c.-à-d. une zone composite large entourant la ville de Nice, qui va jusqu'à Roquebillière et Malaussène vers le nord ainsi qu'à Grasse vers l'ouest ; voir carte B), l'alpin et Saint-Étienne. Les deux premiers systèmes correspondent à ceux décrits par Sumien (2006) pour le nissart et pour le provençal général, respectivement. En revanche, les systèmes pronominaux identifiés sur la base de nos données diffèrent un peu de ceux observés par Dalbera (1985) et cités par Del Giudice (2017). Nous notons aussi une localité, Saint-Étienne, qui semble fonctionner d'une manière distincte des autres localités incluses dans nos données en 
ce qui concerne les pronoms objets ainsi que la présence d'une forme non syncrétique pour le pronom de la troisième personne au datif (3PL.DAT), lor. Enfin, comme c'est le cas pour Dalbera (1991:609), nous observons une forme opaque où le pronom génitif est combiné avec la forme du pronom locatif $i$ (qui est en variation avec la forme du datif $l$ ) pour produire la forme n'in. La distribution géographique de cette forme ne correspond pas à celles de nos trois systèmes pronominaux identifiés ci-dessus.

Enfin, nous identifions trois régions selon les ordres linéaires des systèmes pronominaux, c'est-à-dire, ACC + DAT, DAT + ACC et un ordre linéaire qui varie entre les deux. La distribution selon les ordres linéaires ne correspond pas à celle notée pour les trois systèmes pronominaux. Avant d'analyser nos données, nous décrivons ce qui est connu à propos des principaux dialectes parlés dans les Alpes-Maritimes.

\section{Description des parlers dans les Alpes-Maritimes}

Dans le département des Alpes-Maritimes, situé dans le sud-est de la France, on parle traditionnellement du provençal alpin ou du provençal maritime ainsi que des sous dialectes du ligurien (des parlers gallo-italiques) vers l'est. Ces derniers incluent les dialectes parlés dans des localités de la haute vallée de la Roya, spécifiquement, La Brigue (le brigasc) et Tende (le tendascu) (voir la carte A ci-dessous) alors que le mentonnais serait une variété alpin avec certains traits liguriens (Dalbera, 1994 : 11).

Carte A. - Les Alpes-Maritimes : le réseau d'enquêtes (tiré de Dalbera, 1994).

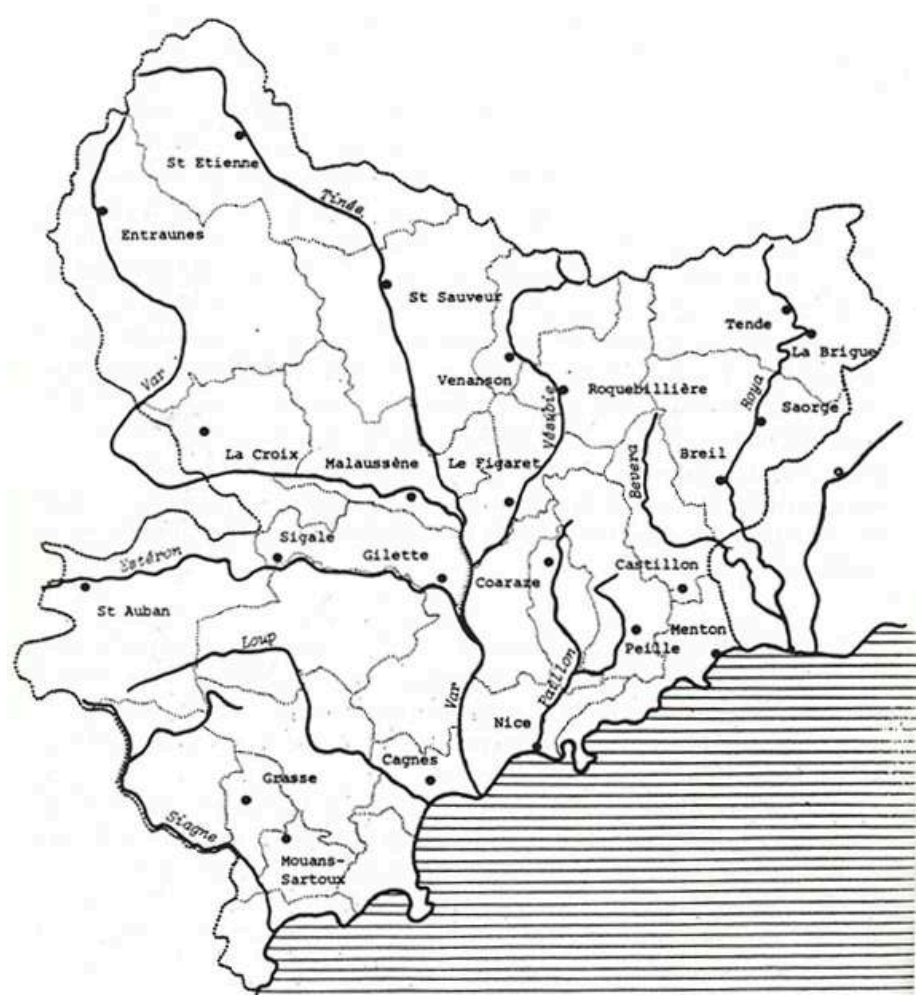

D'après Dalbera, il existe « une petite aire occidentale [...] appartenant au provençal rhodano-méditerranéen, une aire nord-occidentale [...] appartenant au provençal alpin et une aire nissarde » (1994:11), auxquelles s'ajoute le ligurien, d'après la description de ces trois régions dialectales reprise par Del Giudice. Ces auteurs parlent d'abord de 
"deux branches dialectales se démarquant l'une de l'autre» (Del Giudice, $2017: 379$ ). L'une, le ligurien, est confinée à l'est du département tandis que les trois régions dialectales de l'occitan (maritime, alpin, niçois) occupent tout le restant de la zone (ibid.). L'occitan à son tour est divisé, mais d'une manière moins nette, entre « deux manifestations lexicales", soit «le gavot et le provençal maritime» (Dalbera, 1985-1986 : 28, mais voir aussi le point de vue avancé par Del Giudice, 2017)². Étant donné que les dialectes du provençal sont parlés dans la plupart des villages mentionnés, il convient de décrire ses sous-dialectes principaux. Dalbera cite Bouvier (1975) qui décrit l'espace linguistique de l'occitan provençal :

«L'espace dialectal provençal est traversé par deux grands axes qui se recoupent: l'un [...] horizontal allant d'Est en Ouest, l'autre [...] vertical, si l'on peut dire, allant approximativement du Nord vers le Sud en faisant une large boucle. Le premier est bien connu puisqu'il parcourt toute la langue d'oc et distingue des parlers nordoccitans des parlers sud-occitans. [...] Le deuxième est plus spécifique et plus récent puisqu'il ne date que de la fin du Moyen Âge (et) permet de distinguer parlers alpins et non alpins. » (Dalbera, $1994: 11$ ).

6 Ayant présenté les principaux dialectes de la région, nous examinons maintenant les enquêtes menées par Dalbera pour les Parlers des Alpes-Maritimes (PAM) ainsi que les informateurs utilisés pour recueillir des données morphologiques et phonétiques.

\subsection{Les cahiers PAM et les informateurs choisis pour les enquêtes PAM}

7 Nous analysons des données majoritairement inédites provenant des cahiers PAM. Ces enquêtes ont été faites au cours des années 1972 à 1981. Les cahiers PAM font partie du Thesaurus occitan (THESOc: <http://thesaurus.unice.fr/>), qui est une base de données linguistiques des dialectes occitans. Ces données proviennent de sources orales, spécifiquement d'entrevues, et elles sont le résultat d'enquêtes de terrain dans diverses localités (comme celles de notre étude) à différentes époques (cf. Oliviéri \& BrunTrigaud, 2009; Oliviéri, 2011). Les cahiers de terrain scannés contenant les transcriptions phonétiques PAM (Dalbera, 1994) nous ont été fournis par Michèle Oliviéri. Nous disposons de données pour dix-neuf des vingt-trois localités enquêtées, ce qui nous donne un bon nombre de points de données distribués à travers un réseau relativement dense recouvrant le département des Alpes-Maritimes.

Les phrases dans les questionnaires utilisés contiennent des verbes et des pronoms atones objets (directs et indirects) ou des adverbes pronominaux, spécifiquement les équivalents du pronom génitif du français en et du pronom locatif du français $y$. Chaque questionnaire comprend des séries de phrases sur des thèmes reliés, ce qui faciliterait la compréhension pour l'informateur qui traduit les phrases vers son propre dialecte. Afin de mieux comprendre les réponses, Dalbera utilise aussi des dictionnaires et grammaires ainsi que des textes anciens qui sont pertinents à la région en question (Dalbera, $1994: 13$ ).

9 Nous nous focalisons sur une partie des questionnaires selon la localité, soit PAM A (254 questions) et PAM B (367 questions), qui est « consacré à la mise en évidence du système morphologique verbal»(Dalbera, 1994:18). Pour chacune des localités, Dalbera a employé un des deux questionnaires. 
Dans le tableau A, il y a des informations concernant le nombre de participants pour chaque point d'enquête.

Tableau A. - Liste des points d'enquêtes avec nombre de participants.

\begin{tabular}{|c|c|}
\hline Localité & Informateurs pour chaque localité \\
\hline $\begin{array}{l}\text { 1. Cagnes } \\
\text { PAM A ( } 254 \text { questions) complet }\end{array}$ & Une femme (son âge n'est pas indiqué). \\
\hline $\begin{array}{l}\text { 2. Castillon } \\
\text { PAM A ( } 254 \text { questions) complet }\end{array}$ & Un informateur (ni le sexe ni l'âge ne sont indiqués). \\
\hline $\begin{array}{l}\text { 3. Gorbio } \\
\text { PAM B ( } 367 \text { questions) (pas de } \\
\text { réponses }{ }^{2} \text { ) }\end{array}$ & $\begin{array}{l}\text { Une femme et trois hommes ont été interviewés pour tout le } \\
\text { questionnaire y compris le PAM B, mais il y a une seule réponse } \\
\text { par question (sauf quelques précisions pour quelques mots); } \\
\text { leurs âges ne sont pas indiqués. }\end{array}$ \\
\hline $\begin{array}{l}\text { 4. Grasse } \\
\text { PAM A ( } 254 \text { questions) complet }\end{array}$ & Une femme (son âge n'est pas indiqué). \\
\hline $\begin{array}{l}\text { 5. La Brigue } \\
\text { PAM A ( } 254 \text { questions) complet }\end{array}$ & Une femme (son âge n'est pas indiqué). \\
\hline $\begin{array}{l}\text { 6. Malaussène } \\
\text { PAM A ( } 254 \text { questions) complet }\end{array}$ & $\begin{array}{l}\text { Deux femmes pour quelques phrases (leurs âges ne sont pas } \\
\text { indiqués). }\end{array}$ \\
\hline $\begin{array}{l}\text { 7. Menton } \\
\text { PAM A ( } 254 \text { questions) (pas de } \\
\text { réponse * } 1 \text { ) }\end{array}$ & $\begin{array}{l}\text { Questionnaire enregistré avec deux hommes (leurs âges ne sont } \\
\text { pas indiqués); la transcription phonétique a été revue et corrigée } \\
\text { avec ces deux hommes et un autre. }\end{array}$ \\
\hline $\begin{array}{l}\text { 8. L'Escarène } \\
\text { PAM A ( } 254 \text { questions) complet }\end{array}$ & $\begin{array}{l}\text { Une locutrice et un locuteur (leurs âges ne sont pas indiqués) ; il y } \\
\text { a deux réponses seulement pour quelques phrases. }\end{array}$ \\
\hline $\begin{array}{l}\text { 9. Le Figaret } \\
\text { Pas de PAM A ou B } \\
\text { Trois sections : } \\
\text { « Questionnaire de phrases } \\
\text { complétives }: 41 \text { sur } 41 \\
\text { «Impératives »: } 23 \text { sur } 23 \\
\text { «BUT-CAUSE-CONSEQUENCE- } \\
\text { CONCESSION-TEMPS»: } \\
18 \text { sur } 18\end{array}$ & $\begin{array}{l}\text { Deux locutrices, de } 75 \text { ans et de } 48 \text { ans; cette dernière pour } \\
\text { vérifier quelques phrases. }\end{array}$ \\
\hline $\begin{array}{l}\text { 10. Roquebillière } \\
\text { PAM A (pas de réponse } * 4 \text { ) }\end{array}$ & Une femme de « 70 ans environ ». \\
\hline $\begin{array}{l}\text { 11. Coaraze } \\
\text { PAM A : (pas de réponse } * 13)\end{array}$ & Un locuteur (ni le genre ni l'âge ne sont indiqués). \\
\hline
\end{tabular}




\begin{tabular}{|c|c|}
\hline $\begin{array}{l}\text { 12. La Croix-sur-Roudoule } \\
\left.\text { PAM A : (pas de réponse } *_{5}\right)\end{array}$ & Un locuteur (ni le sexe ni l'âge ne sont indiqués). \\
\hline $\begin{array}{l}\text { 13. Sigale } \\
\text { PAM A (pas de réponse *100) }\end{array}$ & $\begin{array}{l}\text { Trois hommes au total ; une seule réponse pour chaque question; } \\
\text { le locuteur n'est pas indiqué. }\end{array}$ \\
\hline $\begin{array}{l}\text { 14. Sainte-Agnès } \\
\text { PAM B ( } 367 \text { questions) (pas de } \\
\text { réponse *4) }\end{array}$ & $\begin{array}{l}\text { Les premières } 38 \text { questions avec deux réponses; les autres avec } \\
\text { une seule réponse (sauf question } n^{\circ} 240 \text { avec deux réponses); } \\
\text { l'informateur n'est pas indiqué. }\end{array}$ \\
\hline $\begin{array}{l}\text { 15. Saint-Étienne } \\
\text { PAM A (pas de réponse *8) }\end{array}$ & Une femme (son âge n'est pas indiqué). \\
\hline $\begin{array}{l}\text { 16. Saint-Sauveur } \\
\text { PAM A (pas de réponse } *_{2} \text { ) }\end{array}$ & $\begin{array}{l}\text { Deux femmes au total ; leurs âges ne sont pas indiqués. Une seule } \\
\text { réponse pour chaque question donc une seule ; l'informatrice (on } \\
\text { n'indique pas laquelle). }\end{array}$ \\
\hline $\begin{array}{l}\text { 17. Tende } \\
\text { PAM A (pas de réponse *17) }\end{array}$ & Un homme (l'âge n'est pas indiqué). \\
\hline $\begin{array}{l}\text { 18. Tourette-Levens } \\
\text { PAM A ( } 254 \text { questions) complet }\end{array}$ & Une femme (une seule réponse pour chaque question). \\
\hline $\begin{array}{l}\text { 19. Venanson } \\
\text { PAM A ( } 254 \text { questions) complet }\end{array}$ & $\begin{array}{l}\text { On a interviewé au total deux femmes et un homme pour tout le } \\
\text { questionnaire (leurs âges ne sont pas indiqués); il y a une seule } \\
\text { réponse pour chaque question; on n'indique pas qui c'est. }\end{array}$ \\
\hline
\end{tabular}

11 Les informateurs choisis par Dalbera pour les enquêtes PAM étaient nés dans la même localité et, dans la plupart des cas, leurs parents en étaient aussi originaires. Ainsi, les informateurs seraient les plus représentatifs de chaque sous-dialecte enquêté. Étant donné que les informateurs avaient une soixantaine d'années à la date des enquêtes de Dalbera (1972 à 1981), les traductions qu'ils nous ont fournies nous donnent une version plus conservatrice du parler local. D’après Dalbera, « la répartition selon le sexe s'est faite spontanément» (1994:19). Ainsi, les femmes et les hommes sont inclus parmi les informateurs, mais pas de façon systématique: selon la localité, les enquêteurs indiquent que des hommes ou des femmes ont été interviewés, mais pour quelques localités il n'y a pas d'information. Spécifiquement, pour six des localités les enquêteurs n'ont pas identifié le genre des informateurs et ainsi nous ne pouvons pas déterminer la répartition entre les sexes (voir le tableau $\mathrm{A}$ ).

Traditionnellement, la majorité des informateurs étaient dans tous les cas des hommes ruraux non mobiles, âgés, (angl. non mobile older rural males NORMS) : « Les informateurs doivent être non mobiles pour s'assurer que leur discours est caractéristique de la région dans laquelle ils vivent. Ils devraient être plus âgés afin de respecter le discours d'une époque révolue. » (Chambers \& Trudgill, $1980: 29$, notre traduction.) Après avoir discuté des dialectes et des participants choisis pour l'étude de Dalbera, nous nous tournons maintenant vers les principaux systèmes pronominaux dans les AlpesMaritimes. 


\section{Les systèmes pronominaux des pronoms objets}

Dans ce qui suit, nous comparons les aires des systèmes pronominaux avec les trois aires linguistiques principales selon les systèmes pronominaux, la forme opaque n'i'n ainsi que les ordres linéaires afin de mettre au clair leur distribution géographique respective (voir Mooney, 2019 pour davantage de détails).

\subsection{Un sommaire des trois systèmes pronominaux (le provençal général et le nissart d'après Sumien, et Saint-Étienne)}

Dans cette section nous examinons les systèmes pronominaux des pronoms objets dans les Alpes-Maritimes afin d'identifier les types principaux, c'est-à-dire les variétés qui jouent un rôle clé, parce qu'elles couvrent géographiquement les différentes parties de la zone examinée et parce qu'elles reflètent les différentes manifestations des systèmes de la zone. Nous comparons ces types avec ceux de Sumien (2006:284).

Dans le tableau B nous examinons les résultats pour Sumien (2006), du moins pour les variétés qu'il désigne comme « provençal général » et « nissart ».

Tableau B. - Les inventaires pronominaux pour le provençal général et pour le nissart d'après Sumien (2006).

\begin{tabular}{|c|c|c|}
\hline & $\begin{array}{l}\text { Le "provençal général» selon } \\
\text { Sumien }(2006: 284)\end{array}$ & $\begin{array}{l}\text { Le «nissart" } \\
\text { Sumien }(2006: 284)\end{array}$ \\
\hline $1 \mathrm{sG}$ & me (m') & mi (m') \\
\hline $2 \mathrm{sG}$ & te $\left(\mathrm{t}^{\prime}\right)$ & $\mathrm{ti}\left(\mathrm{t}^{\prime}\right)$ \\
\hline 3SG.ACC & lo $\left(l^{\prime}\right)$ & lo $\left(l^{\prime}\right)$ \\
\hline 3SG.F.ACC & la (l') & la (l') \\
\hline 3.N.ACC & 0 & 0 \\
\hline 3SG.DAT & li & li \\
\hline Réfléchi & se (s') & si $\left(s^{\prime}\right)$ \\
\hline 1PL & nos & $\operatorname{nos}\left(\mathrm{n}^{\prime}\right)$ \\
\hline Réfléchi (1PL) & se $\left(s^{\prime}\right) /$ nos & si $\left(\mathrm{s}^{\prime}\right) / \operatorname{nos}\left(\mathrm{n}^{\prime}\right)$ \\
\hline 2PL & vos & $\operatorname{vos}\left(v^{\prime}\right)$ \\
\hline 3PL.M.ACC & lei (leis) & lu \\
\hline 3PL.F.ACC & li & li \\
\hline 3PL.DAT & li/lor & li/lor \\
\hline
\end{tabular}




\begin{tabular}{|l|l|l|}
\hline Réfléchi & se (s') & si (s') \\
\hline
\end{tabular}

Dans le tableau C nous comparons nos résultats pour le maritime élargi, l'alpin et SaintÉtienne. Nous constatons que les systèmes se distinguent principalement par les formes de la $1 \mathrm{sG}$ et de la $2 \mathrm{sg}$ ainsi que par l'existence ou non d'une forme non syncrétique pour la 3PL.DAT (lur ou lor) qui est observée dans la localité de Saint-Étienne.

Tableau C. - Une comparaison des inventaires pronominaux de l'alpin, du maritime élargi et de Saint-Étienne.

\begin{tabular}{|c|c|c|c|}
\hline & $\begin{array}{l}\text { La transcription } \\
\text { phonétique de nos } \\
\text { données pour l'alpin }\end{array}$ & $\begin{array}{l}\text { La transcription } \\
\text { phonétique de nos } \\
\text { données pour le maritime } \\
\text { élargi }\end{array}$ & $\begin{array}{l}\text { La transcription } \\
\text { phonétique d'après nos données } \\
\text { pour Saint-Étienne }\end{array}$ \\
\hline $1 \mathrm{sg}$ & {$[\mathrm{me}][\mathrm{m}]$} & {$[\mathrm{mi}][\mathrm{me}]^{3}[\mathrm{~m}]$} & {$[\mathrm{mi}][\mathrm{m}][\mathrm{me}]$} \\
\hline $2 s \mathrm{G}$ & {$[\mathrm{te}][\mathrm{t} \mathrm{t}][\mathrm{t}]$} & {$[\mathrm{ti}][\mathrm{ti}][\mathrm{t}]$} & {$[\mathrm{te}][\mathrm{t}]$} \\
\hline 3SG.ACC & $\begin{array}{l}{[\mathrm{lu}][1] \quad([\mathrm{le}] \text { parfois }} \\
{[\mathrm{u}] /[\text { [ow }]}\end{array}$ & [lu] [l]) & {$[\mathrm{lu}][\mathrm{u}][\mathrm{l}]$} \\
\hline 3SG.F.ACC & [la] [a] & {$[1 \mathrm{a}]^{4}$} & [la] \\
\hline $3 . \mathrm{N}$ & {$[\mathrm{u}][\mathrm{ow}]$} & & [vu] \\
\hline 3SG.DAT & [li] [i] [j] & [li] [i] & {$[\mathrm{li}]$} \\
\hline Réfléchi & [se] [s] & [si] & [si] \\
\hline 1PL & [nuz] & [nu] [nuj] [nuz] [n] & $\begin{array}{l}\text { [nuz] (il y a un seul exemple, } \\
\text { devant voyelle) }\end{array}$ \\
\hline $\begin{array}{l}\text { Réfléchi } \\
(1 \mathrm{PL})\end{array}$ & [si] & [si] [s] & [si] \\
\hline $2 \mathrm{PL}$ & [vuz] & {$[\mathrm{vu}][\mathrm{v}][\mathrm{vi}]$} & [vu] [vuz] [vus] \\
\hline 3PL.M.ACC & [le] [lej] [lej] [lj] & {$[1 \varepsilon j][l i]$} & [lus] \\
\hline 3PL.F.ACC & - & [li] & (pas d'exemple) \\
\hline 3PL.DAT & {$[\mathrm{li}]$} & [li] & [lur] \\
\hline Réfléchi & {$[\mathrm{se}][\mathrm{s} ə]\left[\mathrm{s}^{\prime} \mathrm{e}\right][\mathrm{si}][\mathrm{s}]$} & [si] [s] & [si] \\
\hline
\end{tabular}

Nous examinons maintenant nos résultats pour chacun de ces systèmes, en commençant par l'alpin, ce qui ressemble à ce que Sumien désigne comme le «provençal général » $(2006: 284)$. Le système que nous désignons ainsi apparait dans les variétés parlées dans les localités suivantes: La Croix-sur-Roudoule, Venanson, 
Sigale, Gorbio, Castillon, Sainte-Agnès, Saint-Sauveur et Menton (voir la carte B qui suit ce paragraphe). Nous incluons le mentonnais, qui est considéré par Oliviéri et Del Giudice comme un dialecte de transition (communication personnelle). Ce système se distingue par les formes suivantes : 1sG [me], 2sG [te] et 3sG.RÉFL [se] ou [si].

Carte B. - Les inventaires pronominaux des Alpes-Maritimes sur la base de nos données (adapté de Dalbera, $1991: 610$ ).

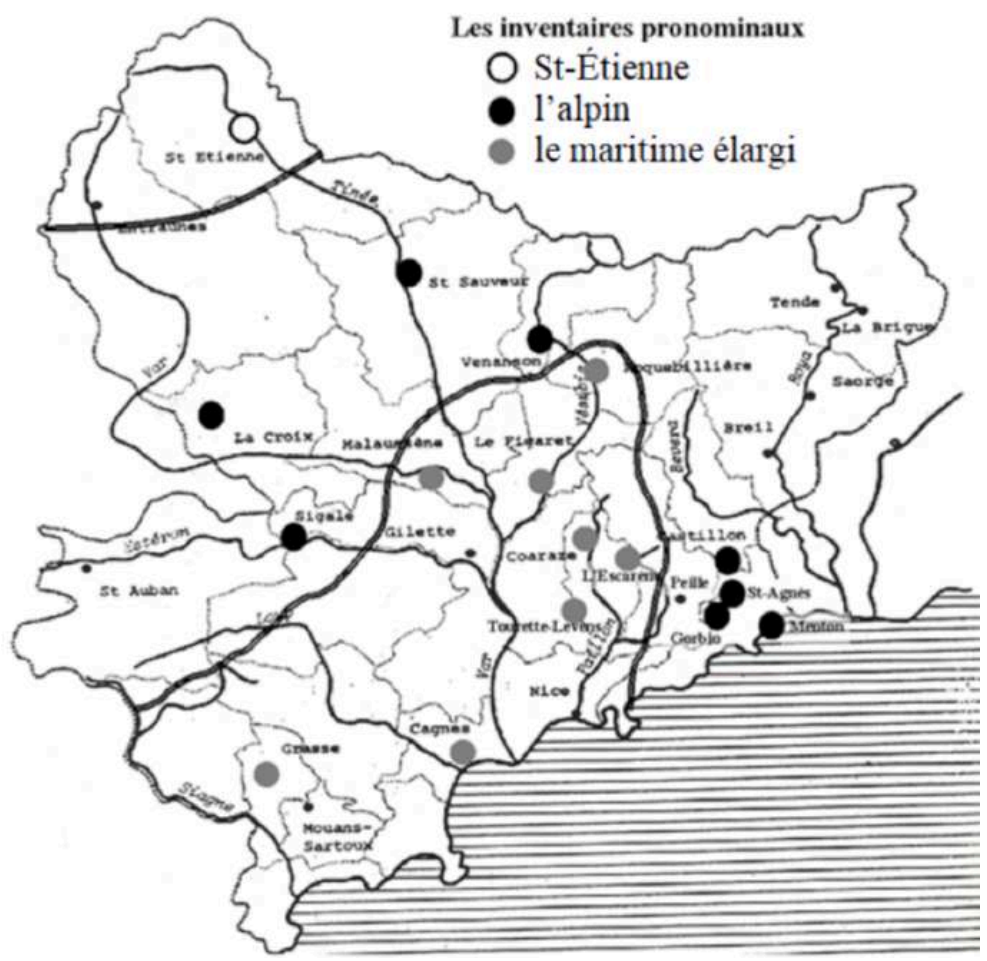

En examinant la carte B nous constatons que la distribution pour l'alpin et pour le maritime élargi ne correspond pas à celle de Dalbera qui parle du provençal rhodanien, du provençal alpin et du nissart. Cependant, ce que nous désignons comme l'alpin comprend des localités proches de la mer telles que Gorbio et Sainte-Agnès ainsi que des localités bien éloignées telles que Venanson et Saint-Sauveur.

En examinant la forme de la 3sG.F.Acc en détail, il y a quelques observations à noter. Pour Sainte-Agnès, nous observons que la forme [a] représente la 3sG.F.ACC :

(1)

$$
\text { [a me kar'ija v'eje ] }
$$

3SG.F.ACC $=1 \mathrm{SG}=$ falloir.3SG.COND voir.INF

«Il me la faudrait voir. »?

( « Il faudrait que je la voie. »)*(Sainte-Agnès, PAM B, ligne 151)

\footnotetext{
* Là où elle apparaît, la quatrième ligne (entre parenthèses) représente la phrase en français à traduire par le participant dans son propre dialecte.
}

L'exemple (1) sert aussi à illustrer la forme de la 1sG.

Dans le questionnaire employé pour obtenir les formes pronominales, il y a très peu de questions avec la 3sG.F.ACC en français (normalement il n'y en a qu'une) ${ }^{5}$. Ainsi, il y a très peu de questions pour obtenir cette forme dans le dialecte du locuteur. Par conséquent, il est difficile de savoir avec précision si cette forme existe dans le 
parler de l'informateur. Pour la localité de Sigale il n'y a pas d'exemple qui nous permette de répondre à cette question.

Dans l'exemple (2) nous observons la forme de la 2sG utilisée en alpin.

(2)

[te faij'a ban d est'a n mum'ent dam 'ela]

$2 \mathrm{SG}=$ faire.3SG.COND bien de rester un moment avec elle

«Il te ferait bien de rester un moment avec elle."

«Ce serait bien que tu restes avec elle un moment. » (Gorbio, PAM B, ligne 76)

La deuxième colonne, qui représente "le maritime élargi ", comprend les localités suivantes: Cagnes, Malaussène, Roquebillière, Coaraze, Tourette-Levens, Grasse, l'Escarène et Le Figaret (voir la carte $B$ ci-dessus). Ce système ressemble au système pronominal du nissart décrit par Sumien (2006: 284).

23 Le système pronominal du maritime élargi se distingue notamment par les formes [mi], [ti] et [si]. Néanmoins, nous observons aussi des exemples de la forme [me] pour représenter la 1sG. Nous illustrons des exemples de la 1sG en (3) et en $(4)^{6}$.

(3)

[la mi sjew mands'a ]

3SG.F.ACC $=1 \mathrm{SG}=$ être. $1 \mathrm{SG}$.IND manger.PART.PST

« Je me la suis mangée. » (Malaussène, PAM A, Section M, ligne 1)

(4)

[v'aw k'awke k'ow me prumen'a me 'ew ]

aller.1SG.IND quelquefois me=promener avec $3 \mathrm{SG} . \mathrm{M}$

«Je vais quelquefois me promener avec lui. » (Cagnes, PAM A, ligne 140)

24 Nous illustrons la forme de la 2sG en (5):

(5)

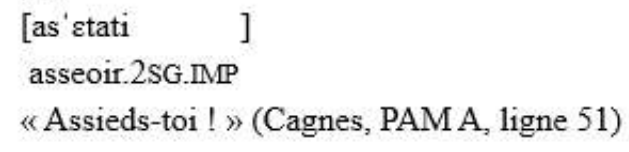

Puisque nous constatons les formes [mi] et [ti] pour le maritime élargi et non pour l'alpin, les formes de la $1 \mathrm{sG}$ et de la $2 \mathrm{SG}$ peuvent donc servir à distinguer ces deux systèmes pronominaux. D'une manière similaire, nous constatons que la forme de la 3PL.M.ACC (ou celle de la 3PL.F.ACC) peut servir à distinguer entre deux dialectes de l'occitan, le languedocien (los (masc.) / las (fém.)) et le "provençal général » (rhodanien et maritime) (lei ou leis pour le masculin ainsi que le féminin) (voir Sumien, $2006: 284$ ). Comme pour l'alpin, toutes les formes du maritime élargi pour la 3sG.DAT sont en variation avec les formes pour le locatif.

La dernière colonne du tableau C représente un système pronominal « isolé », celui de Saint-Étienne. Le système pronominal de Saint-Étienne semble se distinguer du maritime élargi et de l'alpin, car la forme de la 1sG [mi] ressemble au système pronominal du maritime élargi, tandis que l'autre forme de la 1sG [me] ainsi que la forme de la 2sG [te] ressemblent à celui de l'alpin. Néanmoins, nous n'avons pas beaucoup de données pour ces points d'enquête. Enfin, comme c'est le cas pour l'alpin et pour le maritime élargi, nous avons observé une forme distincte pour la 3sG.F.Acc [la] (voir le tableau C ci-dessus). 
Comme nous le notons ci-dessous, Saint-Étienne est aussi la seule localité où nous trouvons une forme distincte [lur] pour la 3PL.DAT (comparer les exemples 6 et 7), tandis qu'il existe du syncrétisme pour le nombre dans toutes les autres localités enquêtées où la forme de la 3sG.DAT, li, est employée également pour représenter la forme de la 3PL.DAT (voir l'exemple 8).

(6)

[k'uma lur d'izun k'uma lur d'izun]*

comment 3PL.DAT=dire.3PL.IND

«Comment ils s'appellent? 》 (Saint-Étienne, «Question complémentaire », question 12)

* La personne enquêtée a donné deux réponses.

(7)

[li puj'a dire sen ke vulj'a ]

"Tu pouvais lui dire ce que tu voulais. "

«Tu pouvais lui raconter n' importe quoi. » (Saint-Étienne, PAM A, ligne 185)

* Le manque de $-s$ désinentiel à Saint-Étienne pour ces formes nous étonne un peu
dans les cahiers PAM. Il s'agit peut-être de formes $3 S G$ interprétées comme une
deuxième personne. De toute façon, ces formes verbales n'affectent en rien les
formes pronominales qui nous intéressent.

(8)

[bẽyl'zw l'aj li agrader'a ply ]

bientôt l'âne 3SG.DAT=plaira plus

«Bientôt l'âne ne leur plaira plus. » (Cagnes, PAM A, ligne 226)

La distinction que nous observons entre les formes pour la 3sG.DAT et la forme pour la 3PL.DAT est notée ailleurs par Dalbera pour Saint-Étienne, mais Dalbera note également cette distinction pour d'autres localités dans le département des AlpesMaritimes, notamment Entraunes et Beuil, qui se trouvent dans la proximité de SaintÉtienne (voir Dalbera, 1991: 605). Ces deux localités ne sont pas incluses parmi les localités pour lesquelles nous avons des données.

Enfin, Saint-Étienne est la seule localité où nous constatons ce qui semble être une forme distincte pour la 3.N.ACC [vu] (exemples 9 et 10).

(9)

[tap'yte vu ]

hacher.2SG.IMP= $3 . \mathrm{N}$

«Hâche-le ! » (Saint-Étienne, PAMA, « Quelques phrases », ligne 24)

(10)

[netj'o vu ]

nettoyer.2SG.IMP=3.N

« Nettoie-le ! » (Saint-Étienne, PAM A, « Quelques phrases », ligne 25)

Pour cette même localité, nous constatons aussi une autre forme qui semble représenter la 3sG.M.Acc :

(11)

[woj lu kunuj ' $\varepsilon$ ] ]

oui 3SG.M.ACC=connaître.1PL.IND

«Oui, nous le connaissons (bien). » (Saint-Étienne, PAM A, ligne 165) 
31 Il faut souligner qu'en ce qui concerne le contexte de la phrase en (11), il semble probable qu'il y ait un antécédent masculin singulier plutôt qu'un antécédent propositionnel (qui donnerait un neutre). Il est possible aussi qu'il n'existe pas de pronom neutre dans les variétés examinées et qu'il s'agisse uniquement de la variation dialectale (Oliviéri, communication personnelle), ou éventuellement, un autre cas de surabondance (Thornton, 2011, 2019). Cela semble être généralement le cas avec SaintÉtienne représentant une exception à la règle. Cependant, dans les exemples (9) et (10) nous avons la forme $v u$, identifiée par Domenge (1999: 66) comme le pronom neutre pour le « provençal ».

Dalbera (1991 : 604) décrit l'existence d'un pronom neutre pour les dialectes parlés dans les Alpes-Maritimes, qu'il contraste avec le pronom de la 3sG.M.Acc. Cependant, en contraste avec nos données, Dalbera (1991) constate une forme neutre à travers une zone plus étendue vers le sud qui comprend aussi Entraunes, Saint-Sauveur, Beuil, La Croix, Sigale, Saint-Auban et Sainte-Agnès.

\subsection{Description de la distribution de la forme opaque n'i'n}

Au-delà des aspects des systèmes pronominaux qui concerne les pronoms individuels et leurs ordres linéaires, nous constatons un exemple d'une forme opaque où le pronom génitif se combine avec la forme du pronom locatif $i$ (qui est en variation libre avec la forme du datifli) pour produire la forme n'i'n. Dans certaines localités des AlpesMaritimes, le pronom génitif [nẽ̃] se combine avec le pronom datif [li] pour produire la forme [njey] (ou [niy] (Dalbera, 1991:609; voir aussi Oliviéri, 1991). La carte C cidessous montre la distribution des localités où cette forme est attestée dans les données des cahiers PAM. 


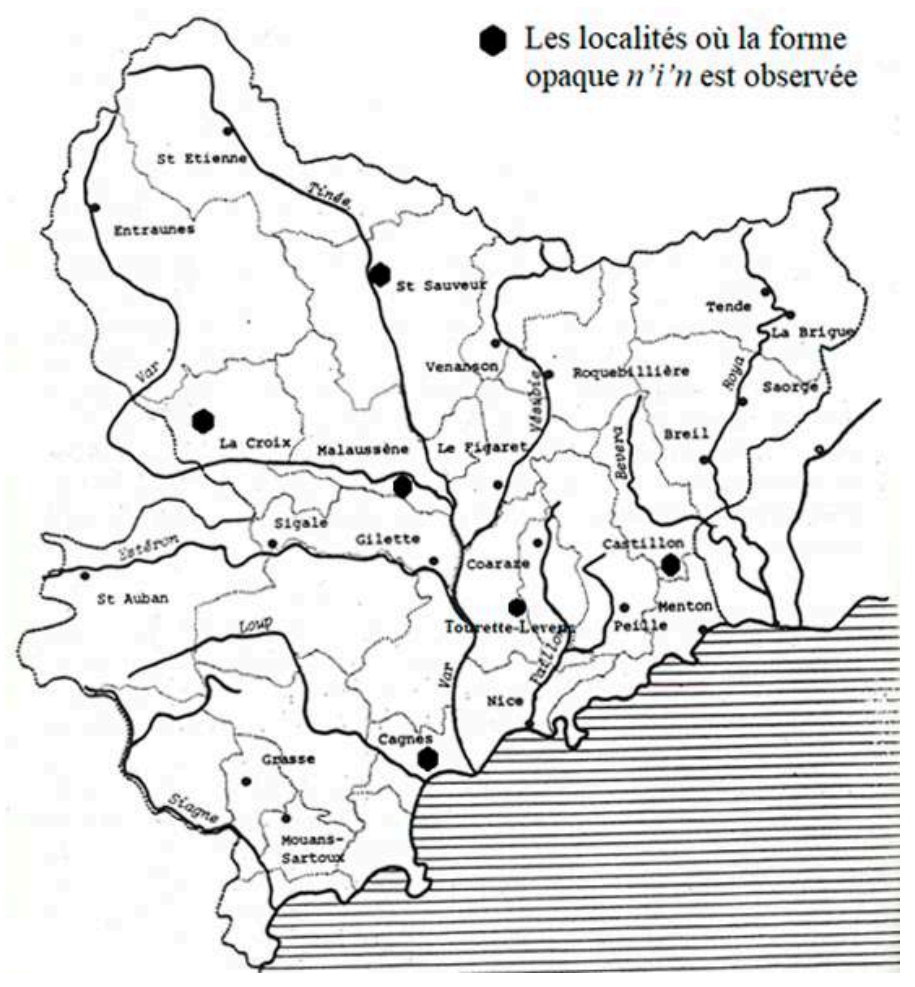

34 Nous constatons qu'il n'y a pas de correspondance entre la distribution de la forme opaque n'i'n et ceux des trois systèmes pronominaux (voir carte B). Néanmoins, les cahiers PAM manquent de données suffisantes pour cette forme opaque pour plusieurs localités et ainsi il est difficile de comparer sa distribution avec celle des autres formes pronominales ${ }^{7}$.

\subsection{La distribution des ordres linéaires des pronoms clitiques selon le cas}

En plus d'examiner les formes morphologiques des pronoms, nous analysons les ordres linéaires des suites de pronoms clitiques. La quantité de données varie pour les différentes combinaisons de la personne grammaticale et du nombre. Dans la région des Alpes-Maritimes, nous constatons que nos données pour les ordres linéaires ressemblent pour la plupart à celles décrites par Dalbera (1991: 609).

Dans nos données, l'ordre linéaire DAT + ACC existe dans trois localités de la partie nordoccidentale (Sigale, Saint-Étienne, La Croix-sur-Roudoule) ainsi que dans une seule localité, Menton, commune située dans la partie orientale près de la frontière italienne (voir la carte D ci-dessous). Pour le mentonnais, Dalbera note aussi l'ordre DAT + ACC. Comme nous le notons, le mentonnais est un dialecte de transition entre l'occitan et le ligurien ${ }^{8}$. Ainsi, on ne s'étonne pas que ce parler puisse avoir une grammaire distincte des autres parlers dans la partie orientale des Alpes-Maritimes. Dans le reste de la partie orientale, nous observons que l'ordre linéaire est ACC + DAT. Cela inclut les localités de Tende et La Brigue, qui appartiennent aux dialectes liguriens (Del Giudice, 2017 : 378, en citant Dalbera, 1985-1986, 1994) ${ }^{9}$. 
Dalbera constate que l'ordre varie entre ACC + DAT et DAT + ACC dans une région intermédiaire entre la région nord-ouest et la partie orientale des Alpes-Maritimes. D'une manière similaire, nous constatons que l'ordre linéaire est généralement ACC + DAT, sauf pour les quatre localités où nous observons l'ordre DAT + ACC (Sigale, Saint-Étienne, La Croix-sur-Roudoule, Menton) ainsi que pour quatre autres localités, Grasse, Malaussène, Saint-Sauveur et Roquebillière, où nous observons que l'ordre varie entre ACC + DAT et DAT + ACC ${ }^{10}$. En contraste avec nos données, pour la localité de Saint-Sauveur, Dalbera constate l'ordre linéaire invariable (DAT + ACC).

Pour Malaussène, nous trouvons dans les exemples en (12) et en (13) que le pronom de la 1sG précède la 3sG.ACC (DAT + ACC), comme c'est le cas en français.

(12)

$[\mathrm{m}$ Jw dj'es tudy'u ]

$1 \mathrm{SG}=3 \mathrm{SG} . \mathrm{ACC}=$ dire $.2 \mathrm{SG} . \mathrm{IND}$ toujours

« Tu me le dis. » (Malaussène, Section I, ligne 1)

(13)

$[\mathrm{m}$ ow az d'itf j'er]

$1 \mathrm{SG}=3 \mathrm{SG} \cdot \mathrm{ACC}=$ avoir.2SG.IND dire.PART.PST hier

« Tu me l'as dit hier. » (Malaussène, Section I, ligne 2)

39 Cependant, pour cette même localité, nous trouvons aussi des exemples de l'ordre linéaire inverse (ACC + DAT) dans les exemples (14) et (15) ${ }^{11}$ :

(14)

[la mi sjew mands'a ]

3SG.F.ACC $=1 \mathrm{SG}=$ être.1SG.IND manger.PART.PST

« Je me la suis mangée. » (Malaussène, Section M, ligne 1)

(15)

[d'i lu $\quad \mathrm{mi}$ ]

dire. 2 SG.IMP $=3$ SG.ACC $=1 \mathrm{SG}$

« Dis-le-moi ! » (Malaussène, Section I, ligne 4) 
Carte D. - Les trois aires linguistiques principales des Alpes-Maritimes selon les ordres linéaires (adapté de Dalbera, 1991 : 610).

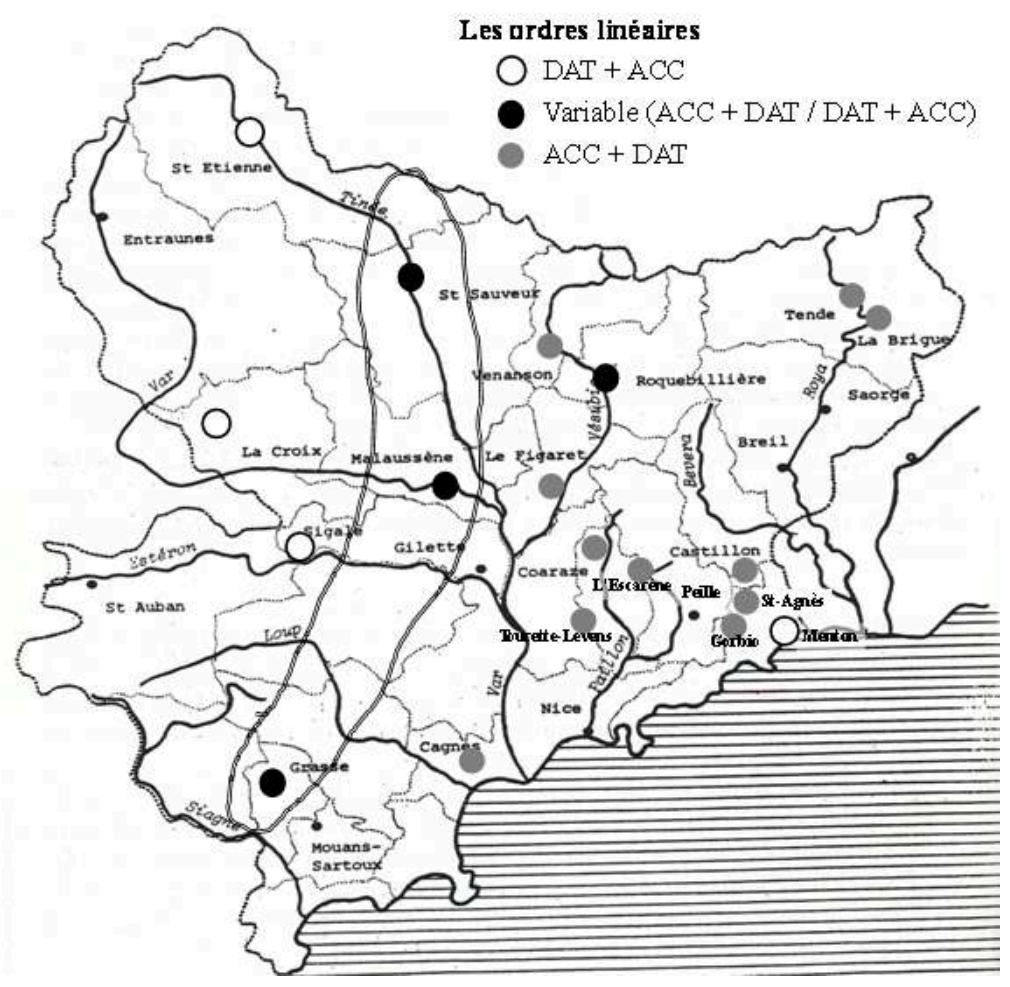

Nous constatons que les aires linguistiques selon les ordres linéaires ne correspondent pas à celles des trois systèmes pronominaux indiqués dans la carte B.

Après avoir examiné la carte des systèmes pronominaux identifiés (voir la carte $\mathrm{B}$ à la section 3.1), nous constatons que chaque système est représenté sous la forme d'un groupement de localités situées d'un côté ou de l'autre d'une ligne tracée sur la carte. De la même manière, les ordres linéaires observés sont représentés en fonction de leur groupement d'un côté ou de l'autre d'une ligne (voir la carte D ci-dessus). Nous examinons ces zones de transition et de possibles interprétations des mouvements dans la section suivante.

\section{Les zones de transition et interprétations possibles des mouvements}

Dans la carte $B$, les limites qui séparent les trois systèmes pronominaux décrits dans cette étude représentent une conceptualisation abstraite de la manière dont les régions dialectales se rencontrent. Cependant, étant donné que la variation réelle est multidimensionnelle, il est plus difficile de la représenter sur les cartes (Chambers \& Trudgill, $1980: 119$ ). La trace d'une isoglosse sur une carte n'implique pas qu'une variable passe à une. Ce fait nous amène à une discussion sur les zones de transition.

Les changements linguistiques, comme ceux que nous constatons pour les ordres linéaires à travers nos trois variétés, sont rarement abrupts (voir la carte $\mathrm{D}$ ci-dessus). D'après Heap (2000: 43-44) cela s'applique en géographie et en diachronie aussi. En d'autres mots, entre deux variétés où le trait $\mathrm{A}$ ou le trait $\mathrm{B}$ est présent, il existe 
normalement au moins une variété où les deux traits sont observés de manière variable (ibid.), ce que Chambers et Trudgill expliquent comme suit :

L'étude de ces variétés intermédiaires mène à établir des « zones de transition » de plusieurs types : des variétés mixtes ( mixed lects ») où on trouve tantôt le trait $\mathrm{A}$ et tantôt le trait $B$, des variétés escamotées («fudged lects») où on trouve une forme intermédiaire entre le trait $\mathrm{A}$ et le trait $\mathrm{B}$, et des variétés embrouillés («scrambled lects») où le trait $\mathrm{A}$ alterne avec le trait $\mathrm{B}$ et avec la forme escamotée intermédiaire. » (1980:125-142; notre traduction) distinguent principalement par les formes de la $1 \mathrm{sG}$ et de la $2 \mathrm{sg}$ ainsi que par la présence ou non d'une forme non syncrétique pour représenter la forme de la troisième personne du pluriel au datif (lur ou lor). Nous avons aussi délimité trois régions dans les Alpes-Maritimes sur la base des ordres linéaires (ACC + DAT, DAT + ACC ou variable entre les deux). Nous avons constaté qu'il n'y a pas de correspondance entre 
ces trois régions et celles que nous avons désignées pour les systèmes pronominaux. D'une manière similaire, nous avons noté qu'il n'y a pas de correspondance entre les localités dans lesquelles la forme opaque n'i’n est observée et les trois régions pour les systèmes pronominaux. Néanmoins, nous manquons de données pour cette forme opaque et il faudrait d'autres questions pour l'obtenir et ainsi mieux discerner la région où elle est observée.

Les trois systèmes pronominaux que nous désignons comme le maritime élargi, l'alpin et Saint-Étienne se distinguent des régions dialectales décrites par Dalbera (et reprises par Del Giudice). Pour deux des phénomènes en question, on peut avancer des interprétations possibles des mouvements des traits de la morphologie pronominale. D'autres données sur les systèmes pronominaux de la région pourront confirmer ou infirmer les analyses et les hypothèses émises ici.

\section{BIBLIOGRAPHIE}

BOUVIER Jean-Claude \& MARTEL Claude, 1975, Atlas linguistique et ethnographique de la Provence, 3 vol., Paris, CNRS.

CHAMBERS Jack \& TRUdGill Peter, 1998, Dialectology, Cambridge, Cambridge University Press.

DALBERA Jean-Philippe, 1985-1986, « Alpes-Maritimes dialectales, essai d'aréologie », Travaux du Cercle linguistique de Nice, $\mathrm{n}^{\mathrm{os}} 10-11$, p. 27-51.

DALBERA Jean-Philippe, 1991, « Les pronoms personnels atones dans les parlers des AlpesMaritimes. Champ et mécanismes de variation », dans D. Kremer (éd.), Actes du XVIII ${ }^{e}$ Congrès international de linguistique et de philologie romanes (Trêves, 1986), Tübingen, Max Niemeyer Verlag, p. 599-613.

DALBERA Jean-Philippe, 1994, Les parlers des Alpes-Maritimes. Étude comparative. Essai de reconstruction, Londres, Association internationale des études occitanes.

DEL GIUDICE Philippe, 2017, Réflexion préliminaire à la réalisation d'un dictionnaire du dialecte niçois, thèse de doctorat en sciences du langage, Université Côte d'Azur.

DOMENGE Jean-Luc, 1999, Grammaire du provençal varois, La Farlede, Association varoise pour l'enseignement du provençal.

HEAP David, 2000, La variation grammaticale en géolinguistique : les pronoms sujet en roman central, Munich, LINCOM Europa.

MOONEY Robert, 2019, La variation de la morphologie pronominale dans les variétés occitanes parlées dans les Alpes-Maritimes, thèse de doctorat, Université Western Ontario.

OLIVIÉRI Michèle, 1991, « La forme n'en nissarde : aperçu sur quelques problèmes morphologiques, syntaxiques et diachroniques ", Travaux du Cercle linguistique de Nice, ${ }^{\text {os }}$ 13-14, p. 61-76. 
OLIVIÉRI Michèle, 2011, « Typology or Reconstruction: The Benefits of Dialectology for Diachronic Analysis ", dans J. Berns, H. Jacobs et T. Scheer (éds), Romance Languages and Linguistic Theory 2009. Selected Papers from ‘Going Romance’ Nice 2009, Amsterdam, John Benjamins, p. 239-253.

OLIVIÉRI Michèle \& BRUN-TRIGAUD Guylaine, 2009, « Le logiciel multimédial Thesaurus Occitan (THESOC) », dans B. Horiot (éd.), La dialectologie hier et aujourd'hui (1906-2006) : actes du colloque, Lyon, Centre d'études linguistiques Jacques Goudet, p. 61-80.

ROSENQVIST Arvid, 1919, «Limites administratives et division dialectale de la France », Neuphilologische Mitteilungen, vol. 20, $\mathrm{n}^{\text {os }}$ 6-8, p. 87-119.

SUMIEN Domergue, 2006, La standardisation pluricentrique de l'occitan. Nouvel enjeu sociolinguistique, développement du lexique et de la morphologie, Turnhout (Belgique), Brepols.

THORNTon Anna, 2011, « Overabundance (Multiple Forms Realizing the Same Cell): A Non-

Canonical Phenomenon in Italian Verb Morphology ", dans M. Maiden, J. C. Smith, M. Goldbach et M. O Hinzeli (éds), Morphological Autonomy: Perspectives from Romance Inflectional Morphology, Oxford, Oxford University Press, p. 358-381.

THORNTON Anna, 2019, « How (Non-)Canonical Is Italian morphology? », dans M. Baerman, O. Bond et A. Hippisley (éds), Morphological Perspectives, Édimbourg, Edinburgh University Press, p. 65-99.

\section{NOTES}

1. Voir aussi la thèse de Mooney (2019).

2. Il aurait été intéressant de tracer des isoglosses sur la carte A, mais nous ne disposons pas d'informations suffisantes pour le faire.

3. Dans la ville de Nice, rarement [me] et seulement dans un contexte particulier (Philippe Del Giudice, communication personnelle).

4. Pour deux localités de cette zone, Coaraze et Malaussène, nous ne trouvons pas d'exemple d'une forme distincte pour la 3sG.F.Acc dans les cahiers PAM.

5. Un des évaluateurs de cet article pour la revue indique que a comme pronom d'accusatif féminin est probablement aussi employé à Peille et à Gorbio.

6. Nous sommes reconnaissants à une des personnes qui a fait une évaluation pour la revue de nous avoir signalé que ces deux formes ([mi] vs [me]) représentent un cas de surabondance (au sens de Thornton, 2011, 2019).

7. Philippe Del Giudice nous indique (communication personnelle) que la forme n'i en existe actuellement à Nice avec une légère différence phonétique.

8. D'après Dalbera (1994:640) et Del Giudice (2017: 379-380), le mentonnais représente un « occitan de transition », c'est-à-dire une variété de transition entre l'occitan et le ligurien.

9. À l'heure actuelle, dans sa langue orale, le niçois de la ville évolue aussi pas mal vers DAT + ACC ; l'ordre varie (Del Giudice, communication personnelle).

10. Pour Roquebillière nous avons plus d'exemples pour l'ordre linéaire ACC + DAT.

11. Lorsque l'ordre de linéaire des pronoms varie entre ACC + DAT et DAT + ACC, il est possible que la nature $d u$ premier segment $d u$ pronom (consonne ou voyelle) joue un rôle. Nous sommes reconnaissants à Philippe Del Giudice de cette observation. Les données à notre disposition ne suffisent pas pour tester cette hypothèse 


\section{RÉSUMÉS}

Nous analysons des données des cahiers PAM (Parlers des Alpes-Maritimes, Dalbera, 1994), provenant d'entrevues orales menées entre 1972 et 1981 . Nous examinons les pronoms atones objets des différentes localités afin d'identifier les variations morphologiques à travers cette région ainsi que des systèmes pronominaux distincts. Nous comparons nos données avec celles des études antérieures, notamment les résultats de Dalbera (1985-1986, 1994) et de Sumien (2006). Nous constatons aussi une forme distincte (non syncrétique) pour le pronom datif à la troisième personne du pluriel, lor, que les locuteurs emploient dans seulement une des localités pour lesquelles nous avons des données, ainsi qu'une forme opaque, n'in, qui représente la fusion du pronom datif avec le pronom génitif dans la partie occidentale des Alpes-Maritimes. Nous comparons la distribution géographique de cette forme opaque n'i'n avec celle des systèmes pronominaux.

We analyse data from the PAM (Parlers des Alpes-Maritimes, Dalbera, 1994) notebooks, which transcribe oral interviews conducted between 1972 and 1981. We examine unstressed object pronouns from different survey points in order to identify morphological variations across this region, as well as distinct pronominal systems. We compare our data with those from earlier studies, in particular the results from Dalbera $(1985-1986,1994)$ and from Sumien (2006). We also observe a distinct (non syncretic) form for the third person dative plural pronoun, lor, only used by speakers in just one of the points for which we have data, as well as an opaque form n'in, which represents the fusion of the dative pronoun with the genitive, in the western part of the Alpes-Maritimes department. We compare the geographic distribution of this opaque form n'in with those of other pronominal systems.

\section{INDEX}

Keywords : pronouns, pronominal systems, Occitan, Alpes-Maritimes

Mots-clés : pronoms, systèmes pronominaux, occitan, Alpes-Maritimes

\section{AUTEURS}

\section{ROBERT MOONEY}

University of Western Ontario

rmooney@uwo.ca

\section{DAVID HEAP}

University of Western Ontario

djheap@uwo.ca 